\author{
Military Technical College \\ Kobry El-Kobbah, \\ Cairo, Egypt.
}

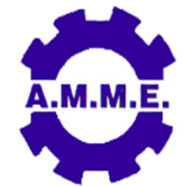

$18^{\text {th }}$ International Conference on Applied Mechanics and Mechanical Engineering.

\title{
MODELING OF $\mathrm{CO}_{2}$ LASER CUTTING PARAMETERS FOR STAINLESS STEEL 316 USING ARTIFICIAL NEURAL NETWORK TECHNIQUE
}

\author{
A. M. El-Wardany ${ }^{1}$, M. A. Mahdy ${ }^{2}$ and H. A. Sonbol ${ }^{3}$
}

\begin{abstract}
Artificial neural networks (ANNs) became one of the most important artificial intelligent tools that have found extensive application in solving many complicated real-world problems. This research presents a new predictive model of $\mathrm{CO}_{2}$ laser cutting of stainless steel 316 using ANN. The aim of this research is to develop an (ANN) model capable to predict the laser cutting process output parameters for certain input variables. The laser beam was used to cut $2 \mathrm{~mm}$ thickness of stainless steel 316 sheet. The input parameters for the neural network are: laser power $(P)$, traverse speed (v), assisted gas pressure(p) and focal plane position (F). The outputs of the neural network model are three most important performance parameters namely: upper kerf width (UKW), lower kerf width (LKW), and the average surface roughness $\left(R_{a}\right)$. The model is based on multilayer feed-forward neural network. The experimentally acquired data is used to train, validate and test the neural network's performance, and special graphs were drawn for this purpose. Finally, this research work would provide a new model based on ANN technique to predict the cutting-edge quality parameters.
\end{abstract}

\section{KEYWORDS}

Laser beam cutting, stainless steel 316, Artificial neural network (ANN), surface roughness $R_{a}$, Upper kerf width, Lower kerf width.

Assistant Lecturer, Modern Academy for Engineering and Tech., Cairo, Egypt.

Dean of Higher Institute for Engineering and Modern Technology Marg, Egypt.

3 Professor, Design and Prod. Engineering Department, Faculty of Engineering, Ain Shams University, Cairo, Egypt. 


\section{NOMENCLATURE}

$\begin{array}{llll}\text { ANN } & \text { Artificial neural network } & \mathrm{P} & \text { Laser power }(\mathrm{kW}) \\ \text { LBC } & \text { Laser beam cutting } & \mathrm{R}_{\mathrm{a}} & \text { Surface roughness }(\mu \mathrm{m}) \\ \text { UKW } & \text { Upper kerf width }(\mathrm{mm}) & \mathrm{v} & \text { Traverse speed }(\mathrm{mm} / \mathrm{min}) \\ \text { LKW } & \text { Lower kerf width }(\mathrm{mm}) & \mathrm{p} & \text { Assist gas pressure }\left(\mathrm{MP}_{\mathrm{a}}\right) \\ \text { ANFIS } & \begin{array}{l}\text { Adaptive neural fuzzy } \\ \text { inference system }\end{array} & \mathrm{F} & \text { Focal plane position }(\mathrm{mm}) \\ & & & \end{array}$

\section{INTRODUCTION}

Laser cutting is a one of the non-traditional cutting process, that has a wide range of applications in different manufacturing processes in industry, due to its capability of cutting with higher quality and cost effectiveness through mass- production rate [1]. It has been proven to be a credible alternative for machining of hard-to-machined materials [2]. The material to be cut is locally melted by the focused laser beam. The molten material is then blown away with the aid of the pressurized assist gas, which flow coaxially with the laser beam, forming a kerf with certain width corresponding to the chosen parameters as shown in Figure 1 [1-3]. The cutting edge produced by laser beam has, always featured parallel grooves similar to those pictured in Ref. [4]. In metal cutting procedures, different types of assist gases are used such as nitrogen, argon, helium, and oxygen. The selection of an appropriate assist gas type or a mixture of gases with a given mixing percentage is necessary to increase the cutting speed and minimizing the cutting cost. [5], however, the use of oxygen as assist cutting gas for cutting mild and stainless-steel increases kerf widths [6].
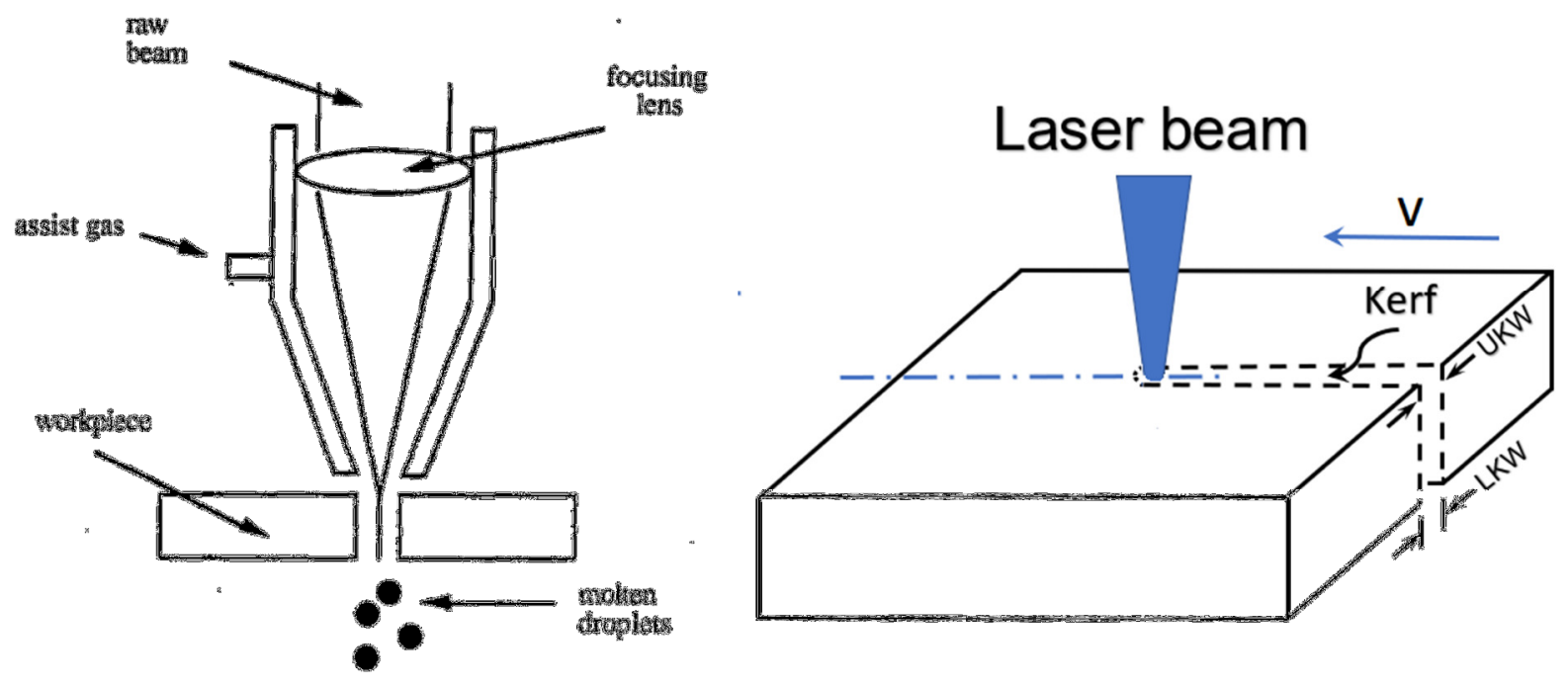

Figure 1. Laser cutting configuration.

The periodic surface structures that are formed on kerf sides after the laser cutting are called striations. Nigade study [7] focuses on striation generation process in laser cutting of steel. In general, striations start from the top of the kerf side and continue to the bottom. Surface roughness formed by these striations is one of the important quality factors in laser cutting process and is to be minimized. 
National and international scholars have done a series of investigations about laser cutting. Typically, the significant parameters of laser cutting quality were investigated. Pocorni et al. [6], reported that, the increase of the material thickness increases the kerf widths and decrease the laser cutting efficiency. Eltawahni et al. [8] has studied effect of laser cutting parameters on operating cost and edge quality of stainless steel sheet with $2 \mathrm{~mm}$ thickness and concluded that the cutting speed is the main factor affecting the upper kerf. In addition, the laser power, nitrogen pressure and focal position have a positive effect on the lower kerf width, while the cutting speed has a negative effect the lower kerf width. Moreover, the roughness value increases as the cutting speed increases and it decreases as the other control parameters increase, the nozzle diameter has no significant effect on the roughness. A reduction of about $71 \%$ in the cutting operating cost could be achieved, if the economical setting is considered.

Intelligent manufacturing is based on the great potential of computer modeling and simulation and information communication technology, and it is composed of intelligent machines and human experts to complete the whole process of product manufacturing. Intelligent manufacturing system can carry out intelligent activities in the manufacturing process, which has the ability of, self-learning, decision, adaptation, and measurement etc. In recent years, the artificial neural network (ANN), had begun to be used by some scholars to predict the laser cutting quality [89]. Zhang et al. [10] analyzed and summarized the importance of the laser cutting roughness prediction and develop ANFIS model to predict the laser cutting roughness with cutting speed, assist gas pressure, cutting width and laser power, as the input variables and surface roughness as output variable. The influence factors of laser cutting quality were studied in the above where provided the basis for solving the problem of laser cutting quality, but few of the laser cutting roughness prediction methods were studied.

The main objective of this research is to develop an (ANN) model, capable to be integrated with intelligent manufacturing systems, to predict the process output parameters for certain input variables. Therefore, it will be the main tool for the application in intelligent manufacturing mode.

\section{EXPERIMENTAL WORK}

In total, 45 samples were produced, and in order to have results that can present an impact on laser cutting industry, all specimens were cut using factors levels around the recommended cutting variables provided by the machine manufacturer. A sheet of stainless steel with standard grade 316 was chosen as work piece material in this research. The sheet dimensions were $400 \times 600 \times 2 \mathrm{~mm}$. At first several trials of laser cutting were performed by varying one of the process parameters at a time to determine the effective range of each parameter. Full cut, keeping the cutting-edge striations, dross and kerf width, to a minimum; were the principles of selecting the working ranges. The experiments were performed according to the design matrix consists of 45 experiments randomly chosen from full factorial design matrix in order to reduce the experimental cost, in addition, the experiments were performed in a random order to avoid any systematic error. Factors, factor levels and factor designations are shown Table 1. A $4.4 \mathrm{~kW} \mathrm{CO}$ laser cutting machine have nozzle diameter $1.5 \mathrm{~mm}$, and focusing lens with focal length of $125 \mathrm{~mm}$ were used to perform 
the cut. A pressurized nitrogen gas was supplied coaxially with the laser beam as an assist gas with different pressures from 1 to $1.5 \mathrm{MP}_{\mathrm{a}}$. Specimens were cut from the sheet plate for each experiment. The test specimen dimension and shape were designed in order to perform a precise measurement of the response parameters. The measurements of upper and lower kerf widths were performed using Mitutoyo optical microscope with an accuracy of $1 \mu \mathrm{m}$, and allow measurements in both the $x$ axis and $y$-axis directions. Three measurements of both kerf widths were performed and the arithmetic average recorded for all specimens.

Table 1. Factors, factor levels and factor designations.

\begin{tabular}{lcc}
\hline Parameters & Unit & Setting values \\
\hline Laser power, $\mathrm{P}$ & $\mathrm{kW}$ & $1,1.25,1.5$ \\
Assist gas pressure, $\mathrm{p}$ & $\mathrm{MP}$ & $1,1.25,1.5$ \\
Focal plane position, $\mathrm{F}$ & $\mathrm{mm}$ & $0,-1,-2$ \\
Traverse speed, $\mathrm{v}$ & $\mathrm{mm} / \mathrm{min}$ & $1000,2000,3000$ \\
\hline
\end{tabular}

The average roughness parameter, $R_{a}$, values were measured using TR200 roughness tester. Three consistent surface roughness values for each specimen were measured at the center of the produced surface, and an average value was calculated for each specimen, Table 2 demonstrates random sample of the experimentally acquired data that were used to train, validate and test the neural network

Table 2. Sample of the experimental data.

\begin{tabular}{cccccccc}
\hline No & $\begin{array}{c}\mathrm{P} \\
\mathrm{kW}\end{array}$ & $\begin{array}{c}\mathrm{F} \\
\mathrm{mm}\end{array}$ & $\begin{array}{c}\mathrm{p} \\
\mathrm{MP}_{\mathrm{a}}\end{array}$ & $\begin{array}{c}\mathrm{v} \\
\mathrm{mm} / \mathrm{min}\end{array}$ & $\begin{array}{c}\mathrm{R}_{\mathrm{a}} \\
\mu \mathrm{m}\end{array}$ & $\begin{array}{c}\mathrm{UKW} \\
\mathrm{mm}\end{array}$ & $\begin{array}{c}\text { LKW } \\
\mathrm{mm}\end{array}$ \\
\hline 1 & 1.25 & 0 & 1 & 2000 & 0.394 & 0.221 & 0.224 \\
2 & 1.5 & 0 & 1.25 & 2000 & 0.409 & 0.231 & 0.245 \\
3 & 1.25 & -2 & 1.25 & 1000 & 0.733 & 0.321 & 0.246 \\
4 & 1.5 & -1 & 1 & 2000 & 0.481 & 0.235 & 0.188 \\
5 & 1.25 & 0 & 1.25 & 1000 & 0.781 & 0.309 & 0.258 \\
6 & 1.25 & 0 & 1.25 & 3000 & 0.578 & 0.18 & 0.173 \\
7 & 1 & -1 & 1 & 2000 & 1.033 & 0.197 & 0.162 \\
8 & 1.25 & -1 & 1.5 & 2000 & 0.61 & 0.264 & 0.209 \\
9 & 1 & -1 & 1.5 & 2000 & 0.835 & 0.251 & 0.155 \\
10 & 1.25 & -2 & 1.25 & 2000 & 0.582 & 0.305 & 0.2 \\
11 & 1.25 & -2 & 1.5 & 2000 & 2.161 & 0.293 & 0.218 \\
12 & 1 & -1 & 1.25 & 2000 & 0.755 & 0.237 & 0.171 \\
13 & 1.5 & -1 & 1.25 & 2000 & 0.634 & 0.281 & 0.186 \\
14 & 1 & -1 & 1.25 & 2000 & 0.697 & 0.272 & 0.197 \\
15 & 1.25 & -2 & 1.25 & 3000 & 1.039 & 0.25 & 0.182 \\
\hline
\end{tabular}




\section{ANN STRUCTURE, TRAINING AND TESTING}

Sufficient training sample size and representation of the training sample for the environment of interest are considered the first and most important step in designing of ANN model. Therefore, for the sake of convenience of comparison, 40 experimental data out of the whole experimental data mentioned above, were used as sample data introduced to the developed network for the purpose of readiness and learning for data prediction while the remaining five experimental data were used to verify the prediction ability for this model. In the developed ANN model, the assist gas pressure, laser power, traverse speed and focal plane position were selected as input parameters for the network, while the output parameter was the upper kerf width, lower kerf width and surface roughness as measures of laser cutting performance which needs to be predicted using ANN model. For our ANN model, the 40-experimental data set, were divided into three sets, namely training data set, validation data set and testing data set with ratios of 70,15 and $15 \%$ respectively. The training data set is used only for learning (i.e., to fit the weights of the network) while, the validation data set is used to minimize over fitting problem that may occur in training process, and the test data set is used to assess the generalization performance of the trained neural network. These three data sets were randomized and then introduced sequentially to the model after the determination of the network structure and suitable training algorithm.

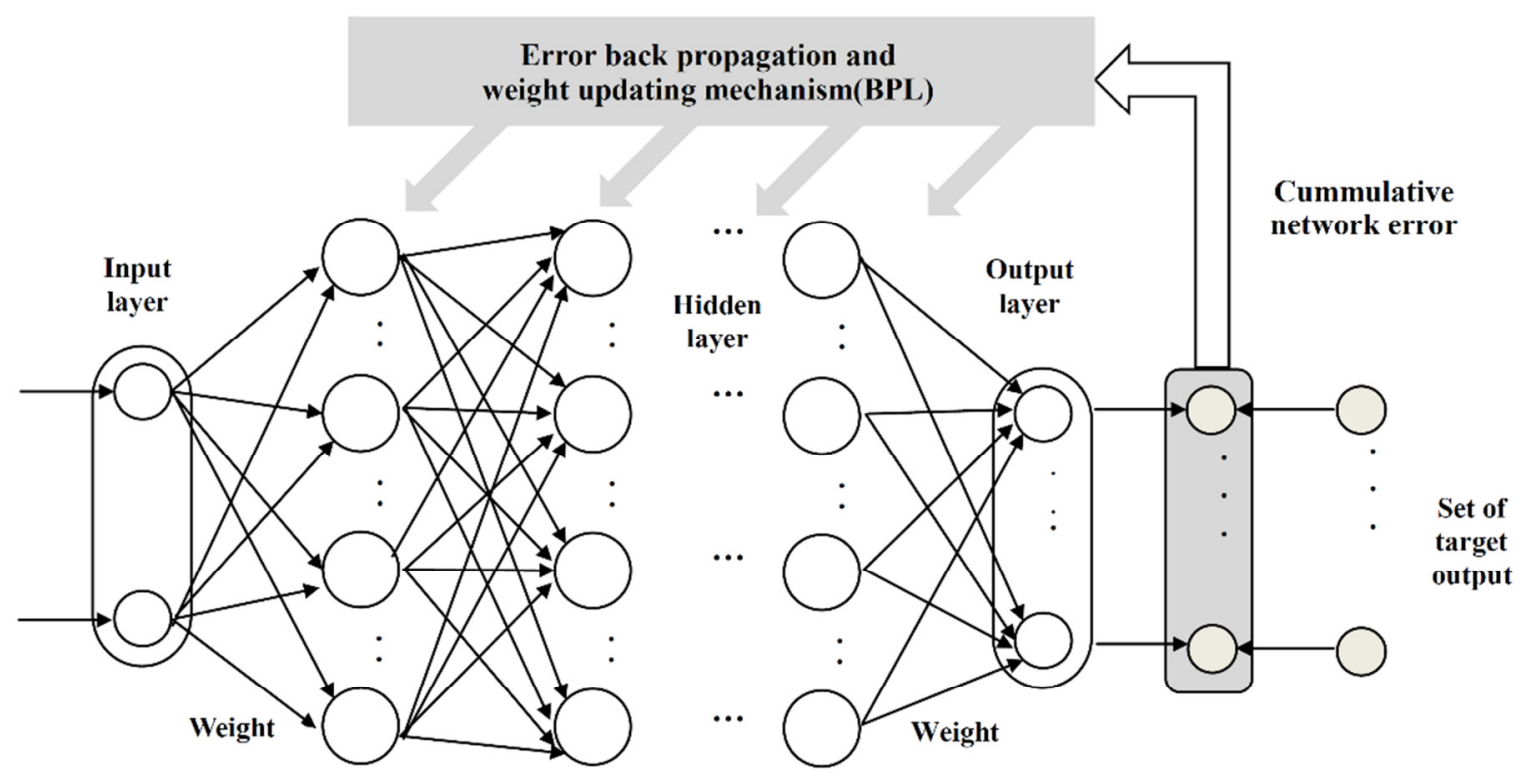

Figure 2. A multi-layer artificial neural network with a back-propagation algorithm

[11].

The feed forward error-back propagation learning algorithm is the most famous procedure for training ANNs. BP is based on searching an error surface (error as a function of ANN weights) using gradient descent for point(s) with minimum error. A typical feed-forward neural network structure with back-propagation algorithm as shown in Figure 2 was adopted in this study. It is most commonly used in the previous researches [9], and includes multiple layers namely input, hidden and output layers with specific number of neurons, initial weights and biases as well as neuron functions. The neuron in each layer performs two functions, summation and 
activation functions respectively to sum the weighted inputs then squash this summation to produce the output. The information is fed and flows between the neurons in the forward direction where each layer receives signals from one neuron before it and passes its output to another subsequent layer. Back-propagation algorithm was used as a training algorithm for feed-forward neural networks, where it would produce the cumulative network error between its final output and the actual output, then this error is back-propagated to adjust the weights values in the whole network mechanism as shown in Figure 2, [12]. One hidden layer was found to be enough in this study and thus the architecture of ANN was a three-layer structure as shown in Figure 3.

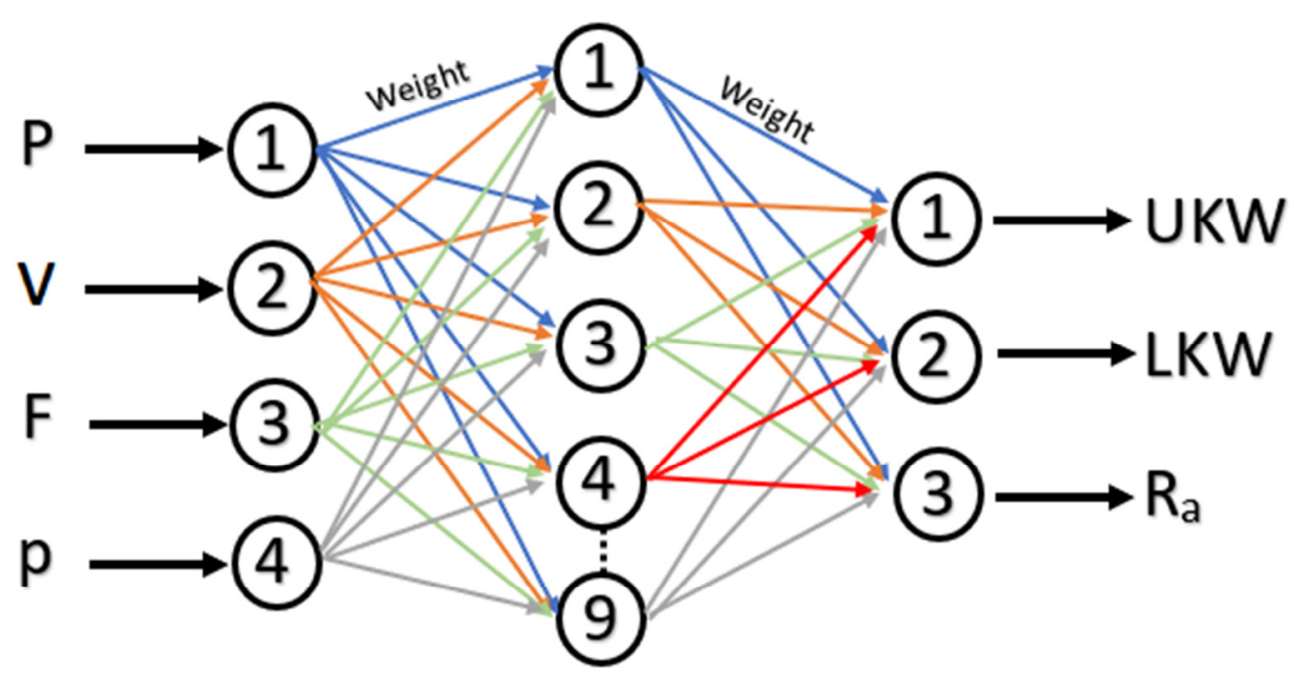

\section{Input laver Hidden layer Output layer}

Figure 3. Architecture of the developed ANN.

The parameters for input layer and output layer had been selected as mentioned previously, while the number of neurons in the hidden layer needs to be optimized. In order to find the optimum number of neurons in the hidden layer, their number was changed iteratively where the mean square error of the network outputs at different number of neurons in the hidden layer were compared as shown in Figure 4.

\section{RESULTS AND DISCUSSION}

Surface roughness and kerf widths would be predicted by the ANN model under the effect of operating parameters, laser power, assist gas pressure, focal plane position and traverse speed. The model had been trained several times until the error between predicted and actual values of the response parameters was minimized. Figure 5 demonstrates the training, validation, and test errors. As shown in the figure, the result is reasonable because of the following considerations: the final meansquare error is small, the validation and test set errors have similar characteristics, and no significant overfitting has occurred by iteration 5 (where the best validation performance occurs). 


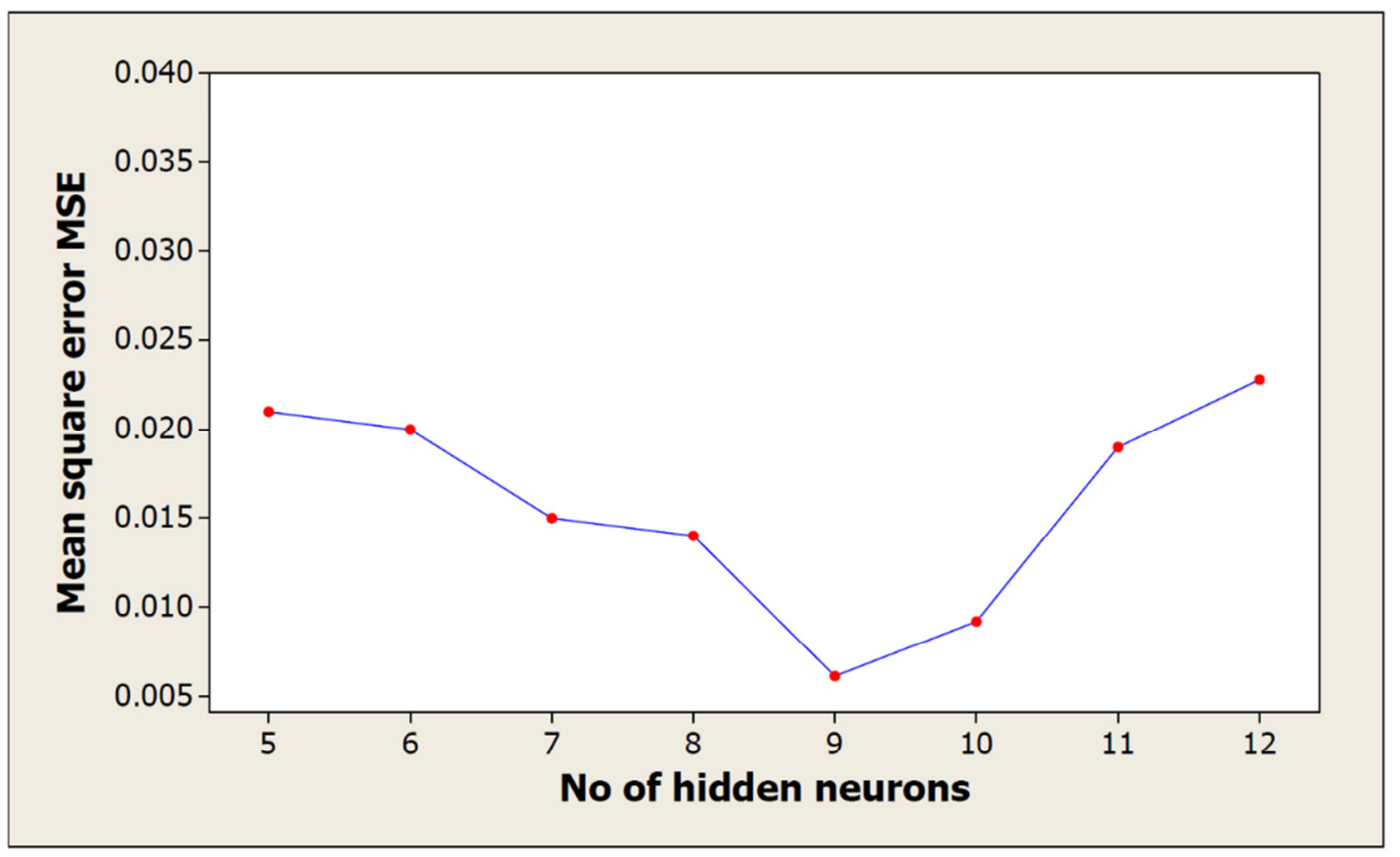

Figure 4. No. of hidden neuron vs. MSE.

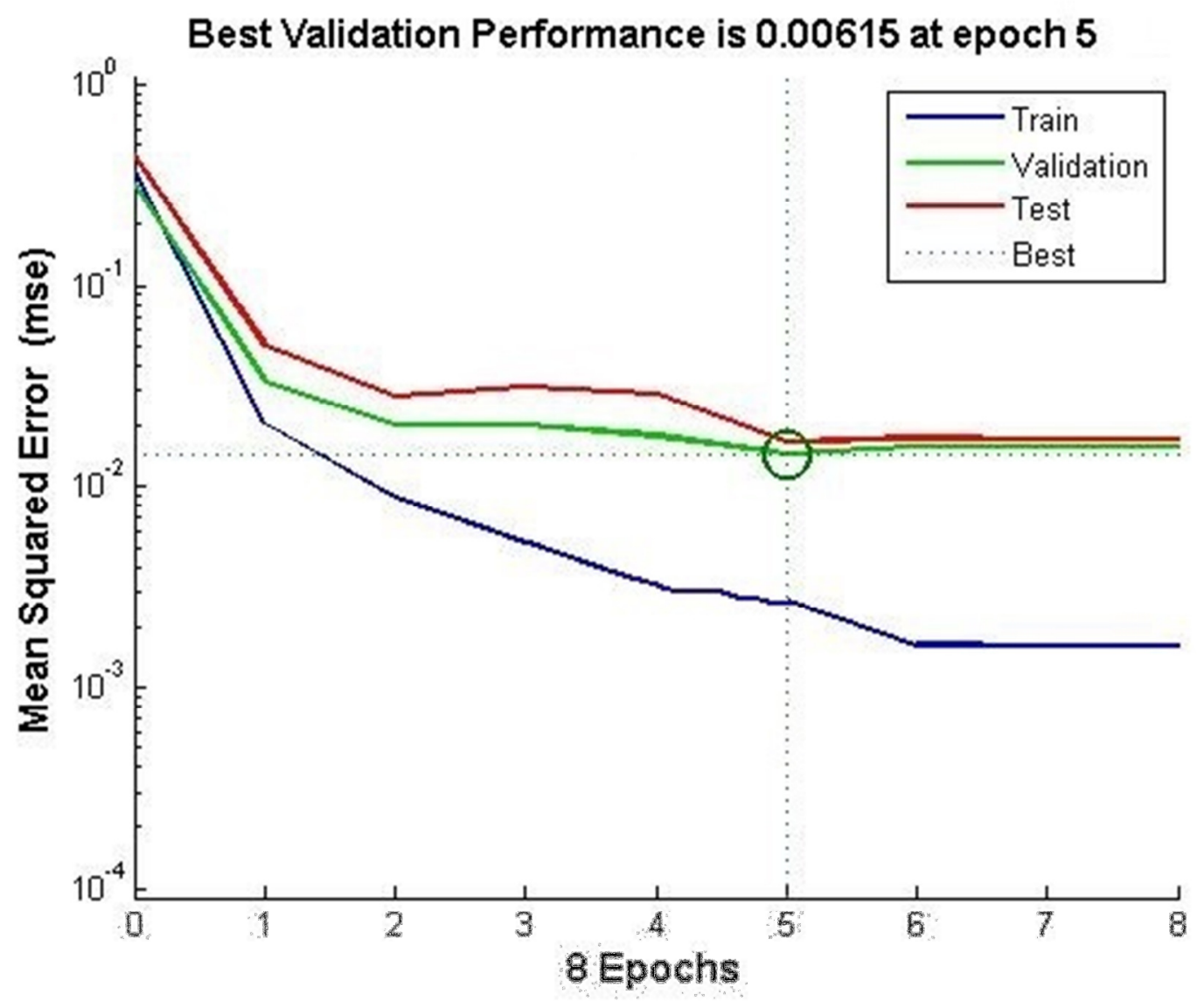

Figure 5. The performance curve for the developed ANN. 
It was also observed that the performance of the proposed ANN in predicting the laser cutting edge quality was satisfactory due to higher values of regression between the predicted and target (actual) outputs during training, testing and validation phases as shown in Figure 6. These regression plots indicated the higher performance of the neural network in predicting responses for any new input variables inside the covered range of this study. In order to check the prediction ability of our ANN model, five experimental data had been used for the purpose of verification. The predicted results from ANN model were compared with corresponding experimental results under the same operating conditions.

Training: $\mathrm{R}=0.99138$

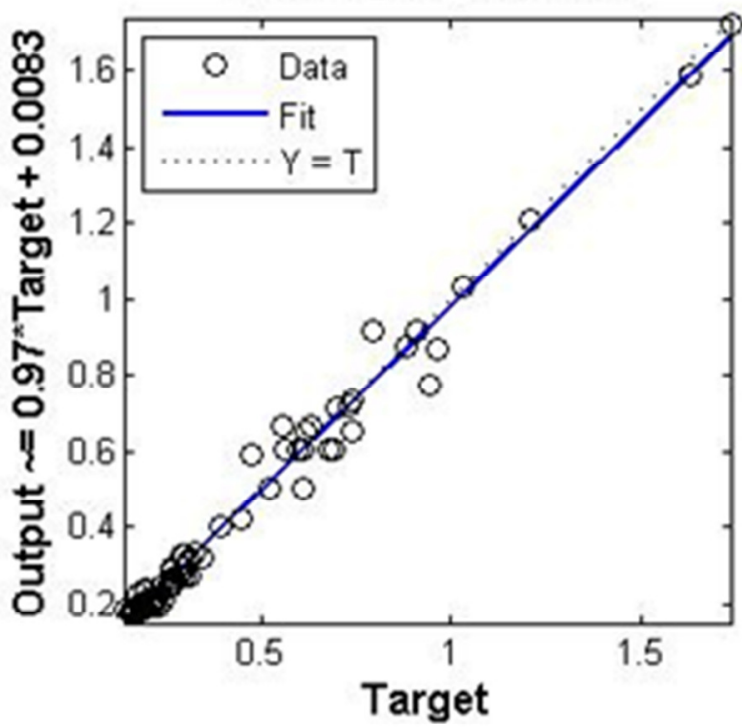

Test: $\mathrm{R}=0.97485$

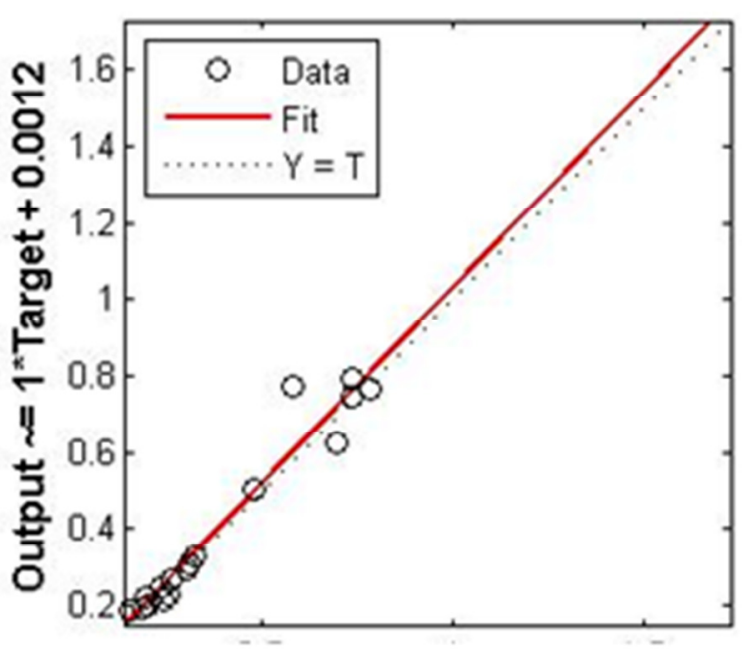

Validation: $\mathrm{R}=0.98432$

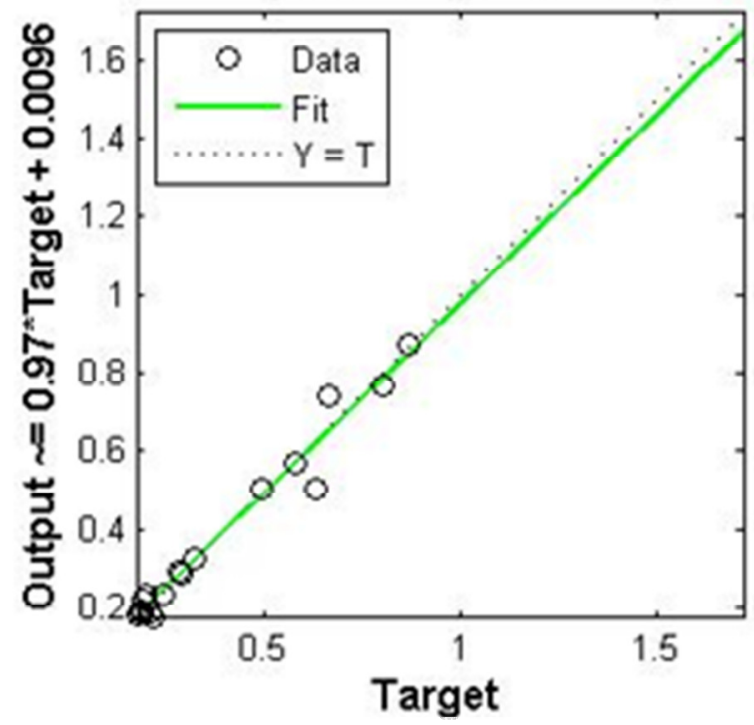

All: $\mathrm{R}=0.9888$

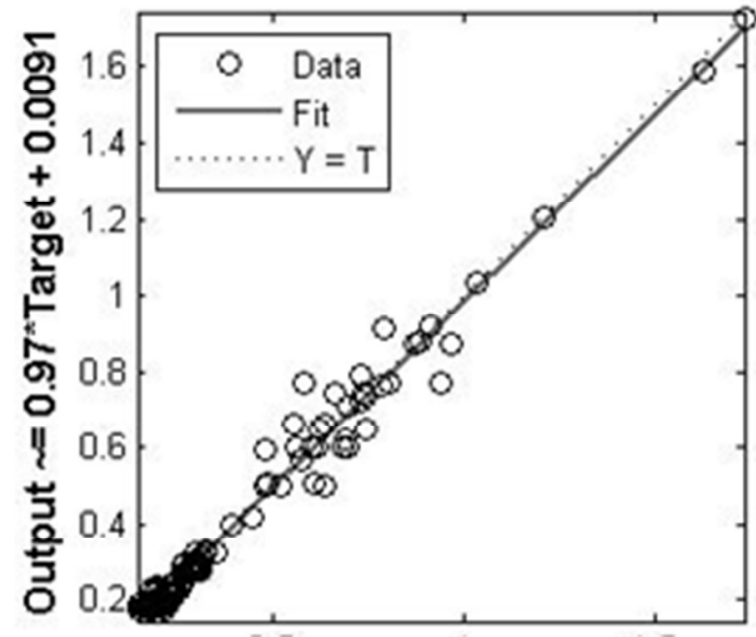

Figure 6. Regression plot for the network performance.

The error percentage of predicted parameters in each verification experiment was calculated as individual for the single hidden layer with 9 neurons and also the prediction error of network for each experiment was calculated as shown in Table 3, from which it was observed that the predicted results were in a good agreement with the experimental results. The average prediction error between the predicted and 
Table 3. Comparison of predicted results by ANN with experimental results.

\begin{tabular}{cccccccccc}
\hline & \multicolumn{3}{c}{$\mathrm{R}_{\mathrm{a}}, \mu \mathrm{m}$} & \multicolumn{3}{c}{ UKW, mm } & \multicolumn{3}{c}{ LKW, mm } \\
NO & Actual & Predicted & Error & Actual & Predicted & Error & Actual & Predicted & Error \\
& result & result & $\%$ & result & result & $\%$ & result & result & $\%$ \\
\hline 1 & 0.814 & 0.745 & 8.38 & 0.223 & 0.221 & 0.69 & 0.167 & 0.176 & -5.45 \\
2 & 0.409 & 0.397 & 2.81 & 0.241 & 0.268 & -11.20 & 0.245 & 0.210 & 13.96 \\
3 & 1.039 & 0.965 & 7.06 & 0.25 & 0.258 & -3.51 & 0.182 & 0.179 & 1.56 \\
4 & 0.755 & 0.712 & 5.58 & 0.237 & 0.267 & -12.85 & 0.171 & 0.181 & -6.11 \\
5 & 0.757 & 0.704 & 6.89 & 0.325 & 0.294 & 9.36 & 0.168 & 0.181 & -8.32 \\
\hline \multicolumn{4}{c}{ Average percentage error for the whole results $=7.859 \%$} & \\
\hline
\end{tabular}

experimental results for the whole compared experiments was found to be $7.859 \%$ which demonstrates the ability of ANN model to predict the response values for any new input values in the covered range with a high degree of accuracy.

\section{CONCLUSIONS}

A novel modeling method for laser cutting performance based on an artificial neural network technique had been presented in this paper. A multi-layer feed-forward network with back-propagation algorithm was adopted for this work. It was found that one hidden layer with 9 neurons can provide a better prediction performance. The average percentage of error for the whole predicted parameters of the model compared to experimental values was $7.859 \%$ which indicates the high accuracy of our model and proves that well-trained neural network models can provide fast, accurate and consistent results. The present work is considered the first attempt in the application of artificial neural network to model a laser cutting. Finally, ANN was found to be a new flexible modeling tool with a high degree of accuracy for linear or nonlinear problems.

\section{FUTURE WORK}

For further development, this model can be extended to include more input and output variables, and also can be integrated with any other intelligent techniques such as genetic algorithm to optimize its structure and hence minimizes the average prediction error.

\section{REFERENCES}

[1] H. El-Hofy, Advanced machining processes, 2005.

[2] Y. Yan, L. Ji, Y. Bao, X. Chen, and Y. Jiang, " $\mathrm{CO}_{2}$ laser high-speed crack-free cutting of thick-section alumina based on close-piercing lapping technique," Int. J. Adv. Manuf. Technol., vol. 64, no. 9-12, pp. 1611-1624, May 2012. 
[3] A. Cekic, D. Begic-Hajdarevic, M. Kulenovic, and A. Omerspahic, " $\mathrm{CO}_{2}$ Laser Cutting of Alloy Steels Using N2 Assist Gas," Procedia Eng., vol. 69, pp. 310315, 2014.

[4] K. Jarosz, P. Löschner, and P. Nies, "Effect of cutting speed on surface quality and heat-affected zone in laser cutting of 316L stainless steel," vol. 149, no. June, pp. 155-162, 2016.

[5] A. N. Fuchs, M. Schoeberl, J. Tremmer, and M. F. Zaeh, "Laser cutting of carbon fiber fabrics," Phys. Procedia, vol. 41, pp. 372-380, 2013.

[6] D. Petring and J. Powell, "The Effect of Laser Type and Power on the Efficiency of Industrial Cutting of Mild and Stainless Steels," pp. 1-6, 2016.

[7] D. B. Nigade and V. S. Jadhav, "Laser Beam Cutting Parameter Optimization for Mild Steel," vol. 3, no. 3, pp. 258-261, 2016.

[8] H. A. Eltawahni, M. Hagino, K. Y. Benyounis, T. Inoue, and A. G. Olabi, "Optics \& Laser Technology Effect of $\mathrm{CO}_{2}$ laser cutting process parameters on edge quality and operating cost of AISI316L," Opt. Laser Technol., vol. 44, no. 4, pp. 1068-1082, 2012.

[9] P. Parandoush and A. Hossain, "A review of modeling and simulation of laser beam machining," Int. J. Mach. Tools Manuf., vol. 85, pp. 135-145, 2014.

[10] Y. Zhang and J. Lei, "Prediction of Laser Cutting Roughness in Intelligent Manufacturing Mode Based on ANFIS," Procedia Eng., vol. 174, pp. 82-89, 2017.

[11] A. A. Rahman and X. Zhang, "ScienceDirect Prediction of Cooling Load for a Standing Wave Thermoacoustic Refrigerator through Artificial Neural Network Technique," vol. 0, no. August, pp. 21-24, 2017.

[12] I. A. Basheer and M. Hajmeer, "Artificial neural networks: fundamentals , computing , design , and application," vol. 43, pp. 3-31, 2000. 